Ann. Génét. Sél. anim., I980, 12 (2), I57-I67.

\title{
Nouvelle estimation de la fréquence du gène pour le noir dans la race ovine Bleu du Maine
}

\author{
C. HAVARD $(*)$, J. J. LAUVERGNE $\left({ }^{* *}\right)$, M. DANZART $\left({ }^{* * *}\right)$ et G. RICORDEAU $(*)$
}

(*) Station d'amélioration génétique des animaux, Centre de Recherches de Toulouse, I.N.R.A., B.P. 12, 31320 Castanet-Tolosan

(**) Département de Génétique animale, Centre national de Recherches Zootechniques, I.N.R.A., 78350 Jouy-en-Josas

(***) Chaive de Mathématique,

Institut National Agronomique, Paris-Grignon, 16, rue Cl. Bernard, 75005 Paris

\section{Résumé}

Une enquête faite en 1978 et 1979 parmi resp. 29 et 49 troupeaux ovins Bleu du Maine de Sarthe et de Mayenne a montré la persistance de l'apparition d'agneaux noirs. Ces agneaux sont de formule $a a, a$ étant récessif par rapport à $A$ wh l'allèle en $A$ gouti responsable de la toison blanche des Bleu du Maine.

Une estimation de la fréquence de $a$ parmi les mâles et les femelles à la naissance $(q)$ et au stade de reproducteurs $(\hat{\alpha})$ a été effectuée par deux méthodes différentes à partir de la fréquence des naissances noires et de celles des mâles hétérozygotes détectés.

Il y avait respectivement en $77 / 7^{8}$ et $78 / 79,5$ I et 85 mâles qui ont donné respectivement I 624 et 270 I agneaux dont respectivement 56 et I o4 étaient noirs. Le nombre des béliers hétérozygotes détectés était respectivement de $2 \mathrm{I}$ et $4 \mathrm{I}$.

En supposant que la fréquence des hétérozygotes femelles est la même dans tous les troupeaux on prend comme estimation la moyenne des estimations par les deux méthodes ce qui donne $\hat{\alpha}_{m}=0,335$ et $0,360 \hat{\alpha}_{f}=0,105$ et $0,1_{12}$ pour les fréquences de $a$ chez les reproducteurs et $q=0,22$ et 0,236 . pour la fréquence à la naissance resp. en $77 / 78$ et $78 / 79$.

En introduisant ces valeurs dans un programme de simulation, on obtient une estimation de I I I agneaux noirs et 44 béliers hétérozygotes détectés pour la deuxième campagne. Ces chiffres sont à rapprocher des deux effectifs observés : respectivement Io4 et $4 \mathrm{I}$ ce qui justifie $a$ posteriori la validité des estimations de fréquence.

La fréquence chez les mâles est significativement plus élevée que chez les femelles, ce qui serait dû à une sélection plus intense chez les mâles que chez les femelles en faveur des hétérozygotes $A$ wh a plus "bleus" que les hétérozygotes $A$ wh $A$ wh. Chez les femelles, si la pression de la sélection artificielle en faveur de 1'hétérozygote est faible, celui-ci pourrait présenter en outre un avantage sélectif (une meilleure prolificité).

C'est grâce à ces avantages naturels et artificiels des hétérozygotes que le gène se maintient à la fréquence élevée que nous observons, malgré l'élimination systématique des noirs de la reproduction. Toutefois, ces fréquences (environ 0,35 pour les mâles et $0, \mathrm{rI}_{\text {pour les }}$ femelles) sont actuellement inférieures à ce qu'elle a pu être dans le passé en races Wensleydale et Bleu du Maine (jusqu'à 0,50 pour les mâles et 0,42 pour les femelles). Cela est dû apparemment à un relachement récent de la pression de la sélection (surtout celle en faveur des mâles hétérozygotes) en zone Bleu du Maine. 
La présence d'un tel gène dans la population Bleu du Maine française présente un intérêt économique (production de 3 p. Ioo d'agneaux en plus à la naissance) pour un désavantage réduit : diminution de 2 p. Ioo de l'efficacité de la sélection.

\section{Introduction}

Il existe en Europe occidentale 3 races ovines qui ont la face et les pattes bleues : les races Blwe face Leicester, Wensleydale en Angleterre, et la race Blew du Maine en France (MASON 1969).

Ce bleuissement est dû à la présence dans le derme de grains de mélanine qui forment écran et permettent une diffraction de la lumière dans le bleu par effet Tyndall (LAUVERGNE et al., I979). Le bleuissement est limité à la face et aux pattes, les poils, même qui poussent dans les zones bleues et la toison restent dépourvus de pigment.

Selon les derniers auteurs cités, et contrairement à l'opinion de LAUVERGNE (1977), la formule génétique de ces races bleues aux loci Agouti et Extension est sans doute $A^{\text {wh }} A^{\text {wh }} E^{+} E^{+}$, c'est-à-dire analogue à celle des races blanches comme le Mérinos ou le Berrichon, avec, pour le bleuissement de la peau, la présence d'un ou plusieurs variants à d'autres loci.

Les races Wensleydale et Blew du Maine se caractérisent en outre par la constante apparition d'agneaux noirs que l'on n'élève pas, tous les reproducteurs étant bleus avec toison blancke. Ce phénomène a été étudié par DRy en Angleterre (I924, I926, I927, I936, I943) et par LAUVERCNE en France (I96I, I962).

Selon LAUVERGNE et al. (I979) ce noir serait dû au gène $a$ récessif par rapport à $A$ wh (blanc ou rouge) au locus $A$ gouti gène dont le maintien dans la population proviendrait d'un élevage sélectif artificiel de l'hétérozygote choisi de préférence à l'hom.ozygote (DRY, I936, I943) car plus "bleu".

Après une première étude de fréquences dans un troupeau privé (LAUVERGNE, I962), l'occasion s'est présentée d'examiner de nouveau ce phénomène d'apparition des agneaux noirs en Bleu du Maine en France. C'est l'objet du présent article.

\section{Matériel et Méthodes}

\section{A. -- Matériel}

Une première enquête a été effectuée en 1978 , (pour la campagne $77 / 78$ ) portant sur 29 élevages adhérant à 1'U.P.R.A. ovine du Maine et localisés en Sarthe et en Mayenne. En 1979, un deuxième sondage portait sur 49 élevages (campagne $78 / 79$ ).

Les paternités ont été obtenues à partir des déclarations de lutte et de misebas fournies par les éleveurs.

Lors du contrôle de performances (pesées à moins de 20 jours), la coloration de tous les agneaux nés a été notée en classant les agneaux en deux catégories :

a) normalement colorés, c'est-à-dire à face et membres bleus, toison blanche (de formule $A^{w h} A^{w h}$ on $A^{w h} a$ ). 
b) noir c'est-à-dire à peau et toison noires (de formule $a a$ ).

On a ainsi pu obtenir la fréquence des agneaux noirs et la fréquence des béliers hétérozygotes décelés.

\section{B. - Estimation de la fréquence génique}

Ce problème d'estimation peut être abordé par deux méthodes différentes

I) en tenant compte du fait qu'un bélier est hétérozygote détecté ou non. détectés.

2) en se basant sur le nombre des descendants noirs des béliers hétérozygotes

La première méthode tient compte du tableau des résultats (où chaque bélier est crédité d'un certain nombre de descendants avec parmi eux des noirs) utilisant l'information à la fois horizontalement et qualitativement (nombre de descendants noirs nul ou non) cependant que la seconde utilise l'information verticalement et quantitativement.

Pour les fréquences de $a$ chez les reproducteurs et les reproductrices on obtiendra ainsi deux estimateurs peu corrélés. Dans l'impossibilité de calculer leur variance on est amené à prendre pour estimateur définitif la moyenne des deux.

On se propose ensuite de tester la validité des résultats obtenus en effectuant des simulations.

\section{I) Notations}

$q$ : fréquence du gène $\underline{a}$ chez les agneaux à la naissance,

$\alpha_{f}$ : fréquence du gène $\underline{a}$ chez les femelles reproductrices (blanches),

$\alpha_{m}$ : fréquence du gène $\underline{a}$ chez les mâles reproducteurs (blancs),

$f$ : fréquence de la naissance d'homozygotes $\underline{a} \underline{a}$,

$b$ : nombre de béliers hétérozygotes détectés,

B : nombre total de béliers,

$n_{i}:$ nombre de descendants du $i$ e bélier,

$\mathrm{N}$ : nombre total de descendants.

Sous l'hypothèse de population infinie panmictique et d'élimination des homozygotes de la reproduction on déduit les relations.

$$
\alpha_{f} \alpha_{m}=f \quad \text { et } \quad \alpha_{f}+\alpha_{m}=2 q
$$

\section{2) Première méthode}

Elle consiste à estimer la probabilité pour un bélier ayant eu $n_{i}$ descendants tous blancs d'être hétérozygote et d'en déduire une estimation du nombre de béliers hétérozygotes non détectés. En employant les notations probabilistes $\mathrm{du}$ tableau I et d'après la formule de Bayes : $\mathrm{P}(\mathrm{A} / \mathrm{B})=\mathrm{P}(\mathrm{B} / \mathrm{A}) \cdot \frac{\mathrm{P}(\mathrm{A})}{\mathrm{P}(\mathrm{B})}$ on obtient :

$$
\mathrm{P}\left(\mathrm{H}_{i} / \mathrm{ND}_{i}\right)=\left[\frac{\mathrm{I}-\alpha_{f} / 2}{\mathrm{I}-f}\right]^{n_{i}} .2 \alpha_{m}=\left[\frac{\mathrm{I}-\frac{f}{2 \alpha_{m}}}{\mathrm{I}-f}\right]^{n_{i}} .2 \alpha_{m}=\mathrm{P}_{i}
$$


TABLEAU I

Notations probabilistes pour la première méthode d'estimation

Probabilistic notations used in the first method of estimation

\begin{tabular}{|c|c|c|}
\hline Probabilité & Notation & Valeur \\
\hline que le bélier $\mathrm{i}$ ayant eu $n_{i}$ desc. tous blancs soit hétérozygote. . . & $\mathrm{P}\left(\mathrm{H}_{i} / \mathrm{ND}_{i}\right)$ & $?$ \\
\hline que le bélier $\mathbf{i}$ soit hétérozygote . . . . . . . . . . . . . . . . . & $\mathrm{P}\left(\mathrm{H}_{i}\right)$ & $2 \alpha_{m}$ \\
\hline que le bélier $\mathrm{i}$ ait ses $n_{i}$ desc. tous blancs . . . . . . . . . . & $\mathbf{P}\left(\mathrm{ND}_{i}\right)$ & $(\mathrm{I}-f) n_{i}$ \\
\hline que le bélier i ait ses $n_{i}$ desc. tous blancs s'il est hétérozygote . . . & $\mathrm{P}\left(\mathrm{ND}_{i} / \mathrm{H}_{i}\right)$ & $(1-\alpha j / 2) n_{i}$ \\
\hline
\end{tabular}

L'espérance du nombre de béliers hétérozygotes non détectés est alors :

$$
\mathrm{A}=\sum_{i=b+1}^{\mathrm{B}} \mathrm{P}_{i}
$$

et l'espérance du taux d'hétérozygotie chez les reproducteurs est :

$$
\mathrm{T}=\frac{\mathrm{B}+\mathrm{A}}{\mathrm{B}}
$$

Partant de la valeur initiale $\mathrm{T}_{0}=\frac{b}{\mathrm{~B}}$ pour estimer le taux d'hétérozygotie chez les mâles reproducteurs on utilise la procédure séquentielle suivante pour obtenir une estimation satisfaisante :

$T_{n}$ étant donné on estime les probabilités $p_{i}$ par $\tilde{p}_{i}^{(n)}$

avec

$$
\tilde{p}_{i}(n)=\left(\frac{\mathrm{I}-\hat{\mathrm{F}} / \mathrm{T} n}{\mathrm{I}-\hat{f}}\right)^{n_{i}} \times \mathrm{T}_{n} \quad \text { où } \quad \hat{f}=\frac{\sum \mathrm{A}_{i}}{\mathrm{~N}}
$$

et l'on en déduit une nouvelle estimation $\mathrm{T}_{n+l}$ définie par

$$
\mathrm{T}_{n+1}=\frac{b+\mathrm{A}_{n}}{\mathrm{~B}} \quad \text { où } \quad \mathrm{A}_{n}=\sum_{i=b+1}^{\mathrm{B}} p_{i}{ }^{(n)}
$$

On obtient de cette matière une suite croissante d'estimateurs par défaut du taux d'hétérozygotie cherché. Cette suite est majorée et elle converge donc vers une limite $\hat{\alpha}_{m}$ qui nous donne la meilleure estimation du taux d'hétérozygotie chez les reproducteurs.

\section{3) Deuxième méthode}

Eille consiste à trouver un estimateur de la fréquence d'hétérozygotie chez les reproductrices à partir des troupeaux dont le mâle est hétérozygote détecté. A l'aide de la formule $\alpha_{f} \alpha_{m}=f$ on en déduit alors une estimation de cette fréquence 
chez les reproducteurs. L'estimation de cette fréquence chez les femelles du $i$ e troupeau est $\frac{2 n_{i r}}{n_{i}} n_{i r}$ étant le nombre de descendants noirs du bélier $i$.

Dans la figure $I$ on donne l'histogramme de la répartition de ces fréquences femelles pour la $2^{\mathrm{e}}$ campagne.

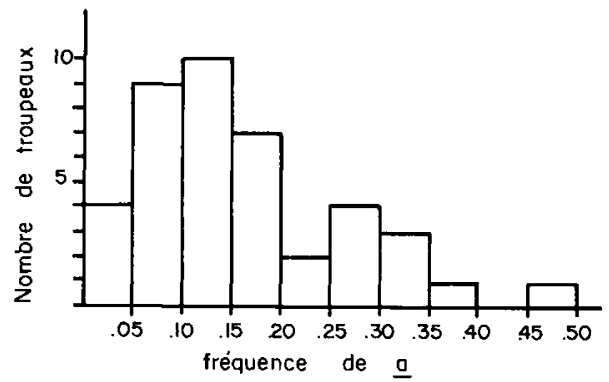

FIG. 1. - Répartition des iroupeaux de la campagne $78 / 79$

selon la fréquence du gène noir a parmi les femelles blanches

Distribution of the flocks of the $78 / 79$ campaign according the frequency

of the black gene a among the white ewes

On remarque le type $B$ de la distribution de ces valeurs qui rappelle les lois de distribution d'une fréquence lorsque la population de départ est subdivisée en petites populations. est :

L'estimation du taux d'hétérozygotie pour l'ensemble des reproductrices

$$
\hat{\alpha}_{f}=\frac{2 \sum_{i=1}^{b} n_{i r}}{\sum_{i=1}^{b} n_{i}}
$$

\section{4) Simulation}

Les tailles de troupeaux étant les tailles réelles on procède à une simulation des naissances en cinq étapes :

I) détermination de l'état hétérozygote ou homozygote du bélier du troupeau considéré.

2) détermination du taux d'hétérozygotie femelle du troupeau.

3) détermination de l'état hétérozygote ou homozygote de chacune des femelles du troupeau considéré.

4) détermination de la couleur noire ou blanche de chaque agneau.

5) passage au troupeau suivant.

Le taux d'hétérozygotie mâle parmi les blancs a pour valeur 0,72 dans cette simulation.

On a effectué roo simulations. 
TABLEAU 2

Effectiț des descendances des béliers Bleu du Maine de l'enquête avec le nombre de naissances noires Size of the progenies of the Bleu du Maine rams of the surveys with the number of black lambs

\begin{tabular}{|c|c|c|c|c|c|c|c|c|c|c|c|c|c|c|c|c|c|}
\hline No bélier & I & 2 & 3 & 4 & 5 & 6 & 7 & 8 & 9 & Io & I I & I 2 & I 3 & I 4 & I 5 & I6 & 17 \\
\hline $\mathrm{Nb}$ descendants. & II6 & II4 & $9 \mathrm{I}$ & 77 & 76 & 68 & 55 & 43 & 40 & 37 & 34 & 33 & 22 & 20 & $\mathrm{I} 8$ & 16 & 15 \\
\hline $\mathrm{Nb}$ noirs . & $\mathrm{I}$ & 14 & 5 & I & I & 6 & 2 & 2 & 4 & $\mathbf{I}$ & 3 & $\mathbf{I}$ & 2 & 2 & 3 & I & I \\
\hline
\end{tabular}

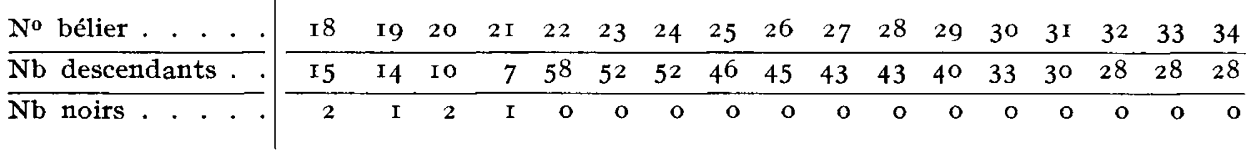

\begin{tabular}{ll|lllllllllllllllll} 
No $^{\circ}$ & bélier . . . . . & 35 & 36 & 37 & $3^{8}$ & 39 & $4^{\mathrm{O}}$ & $4^{\mathrm{I}}$ & $4^{2}$ & 43 & 44 & 45 & 46 & 47 & $4^{8}$ & 49 & $5^{\circ}$ & $5^{\mathrm{I}}$ \\
\hline
\end{tabular}

\begin{tabular}{|c|c|c|c|c|c|c|c|c|c|c|c|c|c|c|c|c|c|}
\hline$\overline{\mathrm{Nb} \text { descendants. }}$. & 27 & 24 & 24 & I7 & I6 & Io & IO & 9 & 9 & 9 & 9 & 5 & 5 & 3 & 3 & 2 & 2 \\
\hline $\mathrm{Nb}$ noirs & o & o & o & 0 & o & 0 & o & 0 & o & 0 & o & 0 & o & 0 & o & 0 & o \\
\hline
\end{tabular}

Campagne $78-79$

\begin{tabular}{|c|c|c|c|c|c|c|c|c|c|c|c|c|c|c|c|c|c|}
\hline No bélier. . . . & I & 2 & 3 & 4 & 5 & 6 & 7 & 8 & 9 & IO & I I & I 2 & I3 & I 4 & $I_{5}$ & I 6 & I 7 \\
\hline $\mathrm{Nb}$ descendants . & 172 & 77 & 76 & 66 & 63 & $6 I$ & 56 & 53 & 53 & 52 & 47 & 44 & 43 & 43 & 43 & 40 & 38 \\
\hline $\mathrm{Nb}$ noirs & 2 & 5 & 3 & $I$ & I & 2 & 2 & 9 & 3 & 5 & 2 & 2 & 4 & 3 & I & 2 & 2 \\
\hline
\end{tabular}

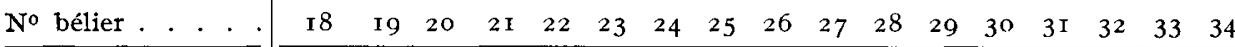

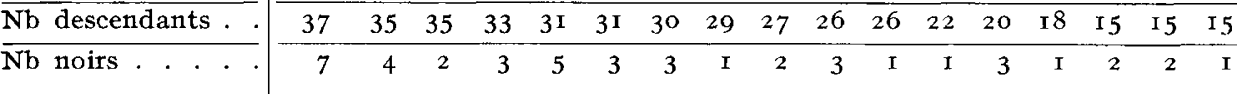

No bélier

$\begin{array}{llllllllllllllllll}35 & 36 & 37 & 3^{8} & 39 & 40 & 4 \mathrm{I} & 4^{2} & 43 & 44 & 45 & 46 & 47 & 48 & 49 & 5 \mathrm{O} & 5 \mathrm{I}\end{array}$

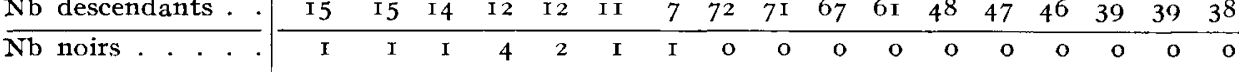

\begin{tabular}{l|rrrrrrrrrrrrrrrrr}
$\mathrm{N}^{\circ}$ bélier . . . . & $5^{2}$ & 53 & 54 & 55 & 56 & 57 & 58 & 59 & 60 & 6 I & 62 & 63 & 64 & 65 & 66 & 67 & 68 \\
\hline $\mathrm{Nb}$ descendants . & $3^{8}$ & $3^{8}$ & 37 & 37 & 34 & 32 & 30 & 30 & 27 & 26 & 25 & 22 & 21 & I9 & I8 & I 8 & 16
\end{tabular}

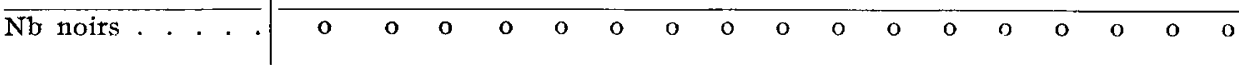

No bélier.

\begin{tabular}{l|rrrrrrrrrrrrrrrrrr}
\hline $\mathrm{Nb}$ descendants. & I6 & I 4 & I 3 & I2 & I 2 & I2 & I0 & 9 & 8 & 8 & 8 & 6 & 5 & 4 & 4 & 3 & 3 \\
\hline Nb noirs . . . & 0 & 0 & 0 & 0 & 0 & 0 & 0 & 0 & 0 & 0 & 0 & 0 & 0 & 0 & 0 & 0
\end{tabular} 


\section{C. - Résultats}

I) données de bases

Les données de départ ainsi qu'un certain nombre de statistiques simples sont présentées dans le tableau 2 .

La figure 2 donne les histogrammes et le tableau 3 les statistiques élémentaires pour les deux campagnes $77 / 78$ et $78 / 79$.

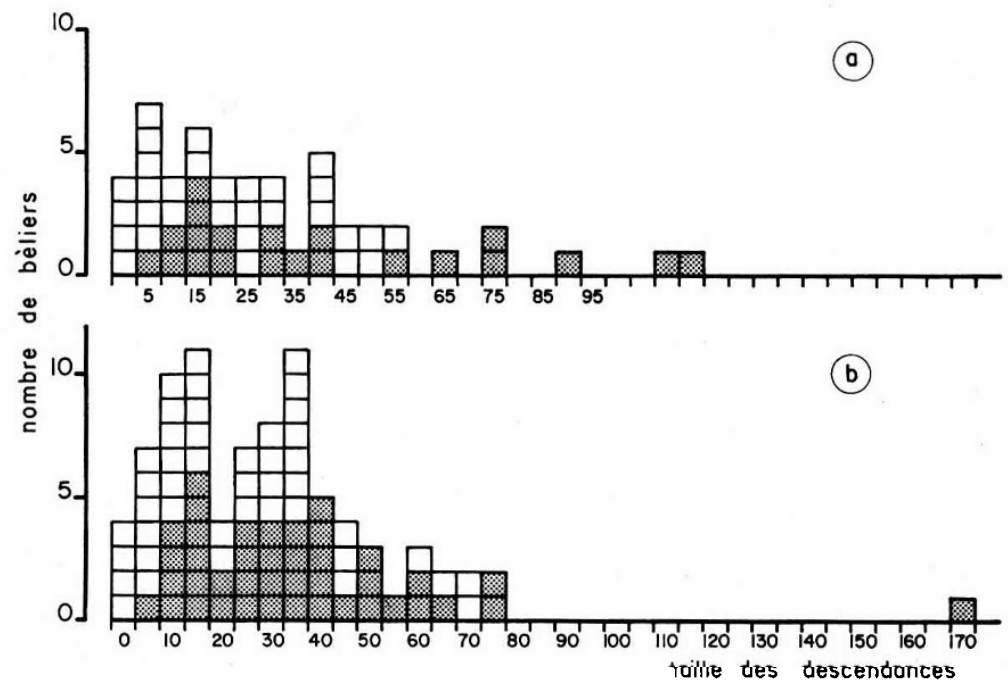

FIG. 2. - Répartition des béliers selon le nombre de leurs descendants.

(Campagnes $77 / 78$ et $78 / 79$ ). (En grisé les béliers hétérozygotes détectés)

Distribution of rams in campaigns $77 / 78$ and $78 / 79$ according the size of their progeny.

(In grey the heterozygote detected rams)

\section{TABLEAU 3}

Résultats des deux enquêtes sur la fréquence des naissances noires en Bleu du Maine Results of two Surveys on frequency of black lambs in Bleu du Maine brced

\begin{tabular}{|c|c|c|c|}
\hline & \multicolumn{2}{|c|}{ Campagnes } & \multirow{2}{*}{$\begin{array}{l}\text { Campagnes } \\
\text { additionnées }\end{array}$} \\
\hline & $77-78$ & $7^{8-79}$ & \\
\hline $\begin{array}{l}\mathrm{Nb} \text { total de béliers } . \quad . \\
\mathrm{Nb} \text { de béliers donnant des noir } \\
\text { \% béliers donnant des noir }\end{array}$ & $\begin{array}{c}5 \mathrm{I} \\
2 \mathrm{I} \\
4 \mathrm{I}, 2 \\
\mathrm{I} 624 \\
56 \\
3,45 \\
32 \\
92 \mathrm{I}\end{array}$ & 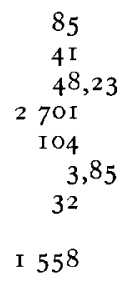 & $\begin{array}{rl} & 136 \\
& 62 \\
& 45,59 \\
4 & 325 \\
& 160 \\
& 3,70 \\
& 32 \\
& \\
2 & 479\end{array}$ \\
\hline
\end{tabular}


TABLEAU 4

Estimation des fréquences génotypiques et des fréquences génétiques par les deux méthodes proposées

Estimation of genotypic and genic frequencies for a with the two proposed methods

\begin{tabular}{|c|c|c|c|c|c|c|c|}
\hline \multirow[b]{2}{*}{ Paramètres } & \multirow[b]{2}{*}{$\begin{array}{l}\text { Sym- } \\
\text { boles }\end{array}$} & \multicolumn{3}{|c|}{ Campagne $77 / 7^{8}$} & \multicolumn{3}{|c|}{ Campagne $78 / 79$} \\
\hline & & $\begin{array}{l}\text { rere } \\
\text { mé- } \\
\text { thode }\end{array}$ & $\begin{array}{c}2^{\mathrm{e}} \\
\text { mé- } \\
\text { thode }\end{array}$ & $\begin{array}{l}\text { moyen- } \\
\text { ne }\end{array}$ & $\begin{array}{l}\text { I ere } \\
\text { mé- } \\
\text { thode }\end{array}$ & $\begin{array}{c}2^{\mathrm{e}} \\
\text { mé- } \\
\text { thode }\end{array}$ & $\begin{array}{l}\text { moyen- } \\
\text { ne }\end{array}$ \\
\hline $\begin{array}{l}\text { Taux d'hétérozygotie chez } \\
\text { les reproducteurs . . . . }\end{array}$ & $2 \hat{\alpha}_{m}$ & 0,76 & 0,58 & 0,67 & 0,87 & 0,57 & 0,72 \\
\hline $\begin{array}{l}\text { Fréquence du gène } a \text { chez les } \\
\text { reproducteurs } . \quad . \quad . \quad . \quad .\end{array}$ & $\hat{\alpha}_{m}$ & 0,38 & 0,29 & 0,335 & 0,435 & 0,285 & 0,36 \\
\hline $\begin{array}{l}\text { Fréquence du gène } a \text { chez les } \\
\text { reproductrices . } . . \\
\end{array}$ & $\hat{\alpha}_{f}$ & $0,09 \mathrm{I}$ & O, I I 9 & 0,105 & 0,085 & $0, \mathrm{I} 35$ & 0, I I 2 \\
\hline $\begin{array}{l}\text { Taux d'hétérozygotie chez les } \\
\text { reproductrices. }\end{array}$ & $2 \hat{\alpha}_{f}$ & 0,182 & $0,23^{8}$ & 0,210 & 0,177 & 0,27 & 0,224 \\
\hline
\end{tabular}

TABLEAU 5

Estimation $d u$ nombre des agneaux noirs et des béliers hétérozygotes par simulation $\left(2^{\mathrm{e}}\right.$ enquête : $\left.78 / 79\right)$

Estimation of numbers of black lambs and of heterozygotic rams by simulation (in the second survey $78 / 79$ )

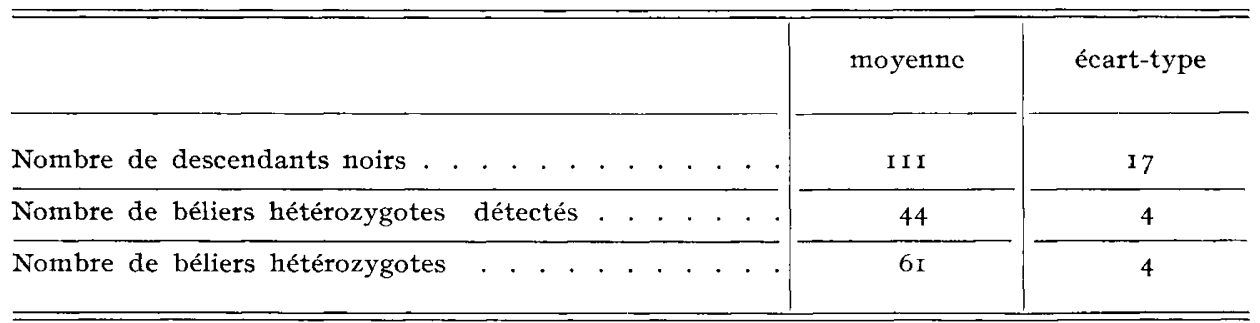

\section{2) Estimation par les 2 méthodes}

Les résultats obtenus par les deux méthodes sont consignés dans le tableau 4.

\section{3) Simulation}

Les résultats obtenus sur roo simulations permettent d'établir le tableau 5 :

Les deux premières lignes du tableau 5 comparées aux résultats réels (IO4 et 4 I) prouvent que les valeurs envisagées pour les taux d'hétérozygotie mâle et femelle sont satisfaisantes.

On prendra donc comme estimation les valeurs moyennes données dans le tableau 6. 


\section{TABLEAU 6}

Estimation des fréquences génétiques de a par les moyennes des deux estimations Estimation of the frequencies of a gene by the average of two estimations

\begin{tabular}{l|c|c|c}
\hline & & campagne $77 / 78$ & campagne $78 / 79$ \\
\hline Fréquence du gène chez les reproducteurs ... & $\hat{\alpha}_{m}$ & 0,335 & 0,360 \\
\hline Fréquence du gène chez les reproducteurs ... & $\hat{\alpha}_{f}$ & 0, I05 & 0, I I 2 \\
\hline Fréquence du gène chez les agneaux à la naissance & $\hat{q}$ & 0,220 & 0,236 \\
\hline
\end{tabular}

\section{Discussion}

D'après le tableau 6 , on voit que les fréquences dans les deux sexes $\alpha_{m}$ et $\alpha_{f}$ ne sont pas différentes d'une année à l'autre mais significativement différentes d'un sexe à l'autre.

On retrouve ainsi l'écart entre les sexes déjà signalé par DRY (I936, I943) en race Wensleydale, puis par LAUVERGNE (1962) en race Bleu du Maine.

L'explication la plus logique, déjà avancée par les précédents auteurs, est que les éleveurs en choisissant leurs reproducteurs prennent de préférence les plus "bleus ". E,t comme les hétérozygotes $A^{w h} a$ seraient en moyenne plus bleus que les homozygotes, ils sont choisis de préférence à ces derniers avec évidemment plus de constance chez les mâles, où le choix est plus grand, que chez les femelles.

Toutefois le niveau auquel s'établit l'équilibre actuel en Bleu du Maine $\left(\alpha_{m}=0,35\right.$ et $\left.\alpha_{f}=0 . \mathrm{II}\right)$ est inférieur à celui constaté en Wensleydale par DRY (I924, I936) aussi bien qu'à celui calculé il y a bientôt vingt ans en France dans le troupeau Chollet-Molines (cf tab1. 7).

\section{TABLEAU 7}

Estimation des fréquences $d u$ gène a dans les deux sexes en races Wensleydale et Bleu du Maine Estimation of frequency of a gene in both sexes in the Wensleydale and Bleu du Maine breeds

\begin{tabular}{|c|c|c|c|c|c|}
\hline \multirow{2}{*}{ Races } & \multirow{2}{*}{ Origine des données } & \multicolumn{2}{|c|}{$q_{a}$} & \multirow{2}{*}{$\begin{array}{c}\text { fréquence } \\
\text { des } \\
\text { noirs }\end{array}$} & \multirow{2}{*}{ Auteurs } \\
\hline & & mâles & femelles & & \\
\hline Wcnsleydale. . & Underley . & 0,5 & 0,416 & 0,208 & DRY (1924) \\
\hline Wenslcydale & Autres troupeaux & 0,5 & 0,314 & 0,157 & DRY (r936) \\
\hline Blou du Maine & Chollet Molines & 0,5 & 0,300 & 0,15 & LAUVERGNE \\
\hline Bleu du Maine & $\begin{array}{l}\text { Enquête BdM } 78 . \\
\text { Enquête BdM } 79\end{array}$ & $\begin{array}{l}0,335 \\
0,360\end{array}$ & $\begin{array}{l}0,105 \\
0,112\end{array}$ & $\begin{array}{l}0,034 \\
0,0385\end{array}$ & $\left\{\begin{array}{c}\text { présent } \\
\text { article }\end{array}\right.$ \\
\hline
\end{tabular}


Cela pourrait provenir du fait qu'en Bleu $d u$ Maine après une mise en veilleuse des activités du Flock-Book, on a, à la reprise, adopté une sélection moins sévère chez les mâles pour le bleu, ce qui fait que seulement ro p. Ioo environ des reproducteurs sont hétérozygotes.

Pour les femelles on note un même relâchement apparent de sélection pour le bleu. Si, en effet, on fixe à 0,36 la fréquence chez les mâles on constate que chez les femelles, s'il n'y a pas de sélection, la fréquence s'établirait à $q=0,22$ valeur observée dans la réalité.

Cette dernière remarque n'est toutefois qu'approximative car on sait depuis Adalsteinsson (I975) que les femelles hétérozygotes $A^{w h} a$ ont un avantage sélectif : elles sont plus prolifiques que les $A^{w h} A^{w h}$ avec environ ro p. Ioo d'agneaux en plus. Avec nos données qui portent sur deux campagnes, et pour lesquelles on n'est pas sûr d'avoir atteint l'équilibre, cet avantage sélectif naturel des femelles est évidemment difficile à mettre en évidence. Cependant que si l'on reprend les données de DRY à Underley, qui s'étagent sur 20 années avec uniquement des mâles hétérozygotes et une sélection phénotypique faible, voir nulle, chez les femelles, on constate que la fréquence d'équilibre du gène chez celles-ci s'établit à 0,40 et non pas à 0,30 comme le donnerait la formule de LAUVVERGNE (rg62) dans le cas ou il n'y aurait aucun avantage sélectif chez les femelles hétérozygotes, ce qui tend à prouver la réalité de cet avantage.

Cet avantage est sans doute économiquement favorable au moins dans l'immédiat, car il procure un excès d'environ 3 p. Ioo d'agneaux par rapport à un troupeau ou toutes les femelles seraient homozygotes pour le blanc. Par ailleurs cela ne nuit pas vraiment à la sélection qui, in fine, s'exerce sur un nombre d'animaux très voisin (99 p. roo) de celui obtenu si toutes les brebis étaient homozygotes blanches.

Cette situation particulière de génétique des populations mérite d'être notée, cependant que les études sont encore nécessaires pour préciser l'action histologique du génotype $A^{w h} a$ pour " bleuir " les animaux qui le portent.

\section{Recu pour publication en août 1980.}

\section{Remerciements}

Ce travail a pu être fait grâce à l'aide et aux encouragements de MM. VIDIs et ForTIN de I'U.P.R.A. Bleu du Maine du Mans et la coopération des éleveurs Bleu du Maine interrogés.

Le manuscrit a été relu par P. MÉRAT Département de Génétique de 1'INRA et Paule RENAUD, Chaire de Mathématique INA Paris-Grignon, qui ont suggéré d'utiles corrections.

\section{Summary}

A new estimation of the frequency of the gene for black in the Bleu du Maine sheep breed

Surveys made in 1978 and 1979 among resp. 79 and 49 Bleu du Maine flocks in the departments of Sarthe and Mayenne have shown that black lambs still appear in the breed. These lambs have a $a$ formula at the $A$ gouti locus, $a$ being recessive to $A^{w h}$ the top allele of the series which gives the white fleece of the breed.

An estimation of the frequency of $a$ among males and females at birth $(q)$ and at the reproductive age $\left(\hat{\alpha}_{m}\right.$ and $\left.\hat{\alpha}_{f}\right)$ has been performed by two methods based on the frequency of blacks at birth and the fequency of known heterozygous rams resp. 
In $77 / 78$ and $78 / 79$ lambings there were resp. 51 and 85 rams used giving resp. I624 and 270 I lambs with resp. 56 and I04 black. The number of detected heterozygous rams was 21 and $4 \mathrm{I}$, resp.

Supposing the same frequency of heterozygous females in all the flocks, the estimation used was the average estimation by the two methods, which gives $\hat{\alpha}_{m}=.335$ and $\cdot 360$ and $\hat{\alpha}_{f}=$ .105 and . I 2 for the frequency of $a$ among the male and female reproducers in resp. 78 and 79 surveys. The frequency among lambs at birth was .220 and .235 resp.

By a program of simulation one get an estimation of I I black lambs and 44 heterozygous sires for the $78 / 79$ campaign, which may be compared to resp. 104 and $4 \mathrm{I}$. This is a fairly good a posteriori verification of the worth of the methods of estimation fot the gene frequencies.

The gene frequency is ligher in male than in female reproducers, which could result from a stronger selection in favour of the heterozygotes $A^{w h} a$ among the males, the heterozygotes having a more intense "blue " coloration. If, among the ewes, the pressure of artificial selection for the heterozygotes is weak, this genotype may have a selective advantage in itself (better prolificacy).

The $a$ gene is probably kept at an equilibrium state at a rather high heterozygotes. Nevertheless, these frequencies (around $.35^{\circ}$ for the rams and. I I for the ewes) are actually inferior to those observed in the past in Wensleydale and Bleu du Maine (up to .5 for the males and .42 for the females). This may be due to a recent relaxing of selection pressure in favour of heterozygous males in the Bleu du Maine zone which has been considered.

The existence of such a gene in the Bleu $d u$ Maine population in France has an economic advantage of about $3 \mathrm{p}$. cent more lambs at birth. On the other hand the culling of black lambs reduces selection efficacy by 2 p. cent.

\section{Références bibliographiques}

Adalsteinsson S., I975. Depressed fertility in Icelandic sheep caused by a single colour gene. Ann. Génét. Sélec. anim., 7, 445-447.

DRY F. W., I924. The genetics of the Wensleydale breed of sheep. I. - The occurence of black lambs -- an examination of flock records. $J$. Genet., 14, 203-21 8.

Dry F. W., I926. Colour inheritance in the Wensleydale breed of sheep. J. Text. Inst., 17, I I 80 - I I 86 ,

Dry F. W., I927. Mendelian breeding with Wensleydale sheep. J. Text. Inst., 18, T 4I5-T 420.

Dry F. W., I936. The genetics of the Wensleydale breed of sheep. II. - Colour fertility and intensity of selection. J. Genet., 33, I 23 -I 24 .

Dry F. W., 1943. The genetics of the Wensleydale breed of sheep. III. - Arithmetical aspects of selection. J. Genet., 45, 265-268.

LAUVERGNe J. J., I96r. Sur le déterminisme génétique de la couleur noire dans la race $\mathrm{B} l e u$ du Maine. Ann. Génét. (Sem. Hôpitaux), 2, 47-52.

LAUVERGNE J. J., I962. Évolution d'une fréquence génique dans la race ovine Bleudu Maine. Ann. Biol. anim. Bioch. Biophys., 2, I I9-127.

LaUVergne J. J., I977. New alleles at the $A$ gouti locus (A) in domestic Ruminants. Xth Int. Pigment Cell Conf., Abstr. no 69.

Lauvergne J. J., Cesbron J., Ortonne J. P., Melchior J., Havard C., i 979 . Gene or genes for skin pigmentation in the sheep: preliminary note. IInd Europ. Worksh. Mam. Melanin Pigm. London, Nov. 21-22, 1979.

Mason I. L., I969. A world dictionary of Livestock types, breeds and varieties. C. A. B. Farnham Royal Bucks. England, i 89-260. 